\title{
A PRELIMINARY LIST OF THE SUBFAMILY Cerambycinae (Coleoptera: Cerambycidae) OF VIETNAM
}

\author{
Cao Thi Quynh Nga*, Khuat Dang Long \\ Institute of Ecology and Biological Resouces, VAST, *cqnga119@yahoo.com
}

\begin{abstract}
Most species of the longhorned beetles are known to damage living trees and attack dead trees or fallen logs and are of economic importance because their woodboring larvae are destructive to plants and freshly cut logs. The paper presented a checlist of publising species of Vietnam longhorn beetle fauna of the subfamily Cerambycinae with local and world distribution provided. Of the total of 259 cerambycid species belonging to 88 genera and 18 tribes, seven diverse tribes are Clytini (74 species), Callichromini (41 species), Cerambycini (35 species), Molorchini (19 species), Callidiopini (18 species), Methiini (13 species) and Cleomenini (11 species).
\end{abstract}

Keywords: Coleoptera, Cerambycidae, Cerambycinae, checklist, distribution, Vietnam.

\section{INTRODUCTION}

The Cerambycinae is a subfamily belonging to the family Cerambycidae, the largest family of Coleoptera. The cerambycids are commonly known as longhorned beetles, longicorns, capricorns, round-headed borers, timber beetles or goat beetles. The representatives of the family Cerambicidae have many varieties of shape, coloration and ornamentation as well as with the body size from $2.5 \mathrm{~mm}$ to $200 \mathrm{~mm}$. The subfamily comprises about 20,000 species occur through almost every part of the world, i.e. from sea level to montane sites as high as 4,200 m elevation. Most species of the longhorned beetles are known to damage living trees and attack dead trees or fallen logs and are of economic importance because their woodboring larvae are destructive to plants and freshly cut logs [8].

Taxonomic interest in the family has been fairly consistent in the past century, however, taxonomic research on the longhorn beetles from Vietnam had been almost absent until recently. The first work of the longhorn beetles in Indochina (including Laos, Cambodia and Vietnam) was published by Fairmaire (1896) [16] and Vitalis de Salvaza (1919) [102], the later authors recorded 348 species from Vietnam. The result of the earliest survey carried out by the Ministry of Agriculture in the period 1967-1968 reported 127 species from
North Vietnam. Mai Phu Quy et al. (1981) [56] also recorded 52 species from the northern part of Vietnam. Recently, from 2005 to 2011, there were 169 the longhorn beetles were recorded from three provinces Quang Tri, Thua ThienHue and Quang Nam, 112 species recorded in the North of central Vietnam, 179 species recorded from four provinces Kon Tum, Gia Lai, Dak Lak and Dak Nong, 2005 (Hoang Vu Tru et al. 2005, 2006, 2009; Ta Huy Thinh et al. 2005) [33-35, 98]. The longhorn beetles of the subfamily Cerambycinae from Vietnam could also be found in several foreign authors as Holzschuh (1989, 1991，1992，1995，1998, 1999, 2003, 2005, 2007, 2009, 2010) [36-46], Niisato (1996, 1998, 2000, 2002, 2004, 2006, 2009, 2011) [58-74], Hayashi (1976a, 1976b, 1981) [27-29], Heffern (2005, 2011) [30-32], Bentanachs et al. (2008) [7], Hua Li-zhong $(2002,2009)[48,49]$. But up to the present time, the taxonomy and systematics of the longhorned beetles of the subfanily Cerambycinae are sudied poorly.

\section{MATERIALS AND METHODS}

The specimens of the longhorn beetles were collected in several surveys orginized by entomologists of Institute of Ecology and Biological Resources (IEBR). All the specimens are deposited in Department of Insect Systematics at IEBR.

The subfamily classification of this study 
follows Gressitt \& Rondon (1970) [25]. The original taxonomy documents in this study mainly refer to Aurivillius (1911, 1912) [2, 3], Bates (1873, 1879) [4, 5], Fabricius (1775) [14], Fairmaire (1895, 1896) [16, 17], Gahan (1906) [21], Pascoe (1856-1859, 1864-1869) [75-85], Lacordaire (1869) [52], Pic (1911, 1922, 1923, 1925, 1933) [86-92] and White (1853, 1855) $[105,106]$. The distribution documents from Vietnam refer to the authors: Abang (2013) [1], Bentanachs (2005, 2008) [6, 7], Hayashi (1976a, 1976b, 1981) [27-29], Heffern (2005, 2007, 2011) [30-32]; Holzschuh (1998, 1991, 1992, 1995, 1998, 1999, 2003, 2005, 2007, 2009, 2010) [36-46], Hudepohl (1990) [50], Hua Li-zhong (2002, 2009) [48, 49], Niisato $(1990,1998,2000$, 20022, 2002b, 2004, 2006, 2009, 2011) [58-74], Vives \& Bentanachs (2007) [103] and Vives \& Mei-ying Lin (2013) [104].

Abbreviations of localities used in this paper are as follows: E: East; N: North; S: South; SW: Southwest; old name of localities used in the literature: Annam = North Central; Cochin= Southern Vietnam; SE: South East and Tonkin $=$ Northern Vietnam.

\section{RESULTS AND DISCUSSION}

This paper presents a list of 259 species in the systematic sequence of the following 18 tribes and 88 genera completing them with the number of the species recorded from Vietnam and in the parenthesis the number of the generic names. We included in the list for every species the page of first citing species for Vietnam, and the distribution of species in Vietnamese provinces shown in the parenthesis.

$\begin{array}{ll}\text { Achrysonini (1): } & 1 \\ \text { Callichromini (11): } & 41 \\ \text { Callidiopini (8): } & 18 \\ \text { Cerambycini (18): } & 35 \\ \text { Cleomenini (5): } & 11 \\ \text { Clytini (9): } & 74 \\ \text { Hesperophanini (3): } & 4 \\ \text { Methiini (7): } & 13 \\ \text { Molorchini (6): } & 19 \\ \text { Mythodini (1): } & 1\end{array}$

$\begin{array}{ll}\text { Obriini (6): } & 11 \\ \text { Prothemini (1): } & 3 \\ \text { Purpuricenini (2): } & 3 \\ \text { Pyrestini (4): } & 7 \\ \text { Phoracanthini (2): } & 5 \\ \text { Rosaliini (2): } & 6 \\ \text { Tillomorphini (1): } & 2 \\ \text { Thraniini (1): } & 5\end{array}$

\section{Systematics}

Tribe Achrysonini Lacordaire, 1869

\section{Bumetopia flavovariegata flavovariegata Aurivillius, 1911}

Bumetopia flavovariegata flavovariegata Aurivillius, 1911: 23.

Heffern, 2011: 29.

Distribution: Vietnam; Indonesia (Borneo, Sulawesi).

Tribe Callichromini LeConte, 1873: Gahan, 1906: 189.

\section{Anubis bipustulatus fimbriatus Bates, 1879}

Anubis bipustulatus fimbriatus Bates, 1879: 412; Gahan, 1906: 222.

Bentanach et al., 2008: 17.

Synonym: Anubis bipustulatus fimbriatus Plavilshikov, 1934.

Anubis bipustulatus f. tripustulatus Plavilshikov, 1927.

Distribution: Vietnam: Annam (Quinhone); India; Laos; Thailand; Malaysia.

\section{Anubis bipustulatus Thomson, 1865}

Anubis bipustulatus Thomson, 1865: Gahan, 1906: 221.

Gressitt \& Rondon, 1970: 165.

Distribution: Vietnam: Annam, S. Vietnam; N. India; China; Taiwan; Laos; Malaysia.

\section{Anubis cyaneus Pic, 1924}

Anubis cyaneus Pic, 1924: Gressitt \& Rondon, 1970: 164.

Bentanach J. et al., 2008: 21.

Synonym: Polyzonus democraticus Podany, 1980. 
Distribution: Vietnam: Annam (Cua Tung, Synonym: Aphrodisium griffithi: Gahan, 1906 Quang Tri); China; Laos.

Distribution: Vietnam: S. Vietnam.

5. Anubis inermis (White, 1853)

Polyzonus inermis White, 1853: 171.

11. Aphrodisium (s. str.) semipurpureum Pic, 1925

Hua Li-zhong, 2002: 194.

Distribution: Vietnam; NE. India; W. Pakistan; Sikkim; Myanmar; Nepan; Laos; S. China; Thailand; Malaysia.

\section{Anubis rostratus Bates, 1879}

Anubis rostratus Bates, 1879: 412; Gahan, 1906: 221.

Gressitt \& Rondon, 1970: 165.

Synonym: Anubis rostratus var. annamitus Pic, 1946.

Distribution: Vietnam: Tonkin, S. Vietnam (Kon Tum); China; Myanmar; Laos; Cambodia.

7. Anubis subobtusus (Pic, 1932)

Polyzonus subobtusus Pic, 1932: Gressitt \& Rondon, 1970: 163.

Bentanach et al., 2008: 30.

Synonym: Polyzonus subobtusus ab. Interruptus Pic, 1946; Polyzonus s.str. subobtusus Podany, 1980; Polyzonus (Polyxonides) thoracicus Podany, 1980.

Distribution: Vietnam: Tonkin, Saigon.

8. Anubis unifasciatus Bates, 1879

Anubis unifasciatus Bates, 1879: 412.

Bentanach et al., 2008: 32.

Distribution: Vietnam (Phan Thiet); Myanmar;

Laos; Thailand.

\section{Anubis viridicollis Pic, 1932}

Anubis viridicollis Pic, 1932: Podany, 1980: 223.

Bentanach et al., 2008: 33.

Distribution: Vietnam: Tonkin (Dong Van); Laos; Cambodia; Thailand.

10. Aphrodisium (Opacaphrodisium) griffithi (Hope, 1841)

Callichroma griffithi Hope, 1841: Gressitt \& Rondon. 1970: 144.

Gressitt \& Rondon, 1970: 144.

Aphrodisium (s.str.) semipurpureum Pic, 1925: 18.

Gressitt \& Rondon, 1970: 145.

Distribution: Vietnam; Laos.

12. Aphrodisium delatouchei Fairmaire, 1886

Aphrodisium delatouchei Fairmaire, 1886: Podany, 1971: 282.

Hua Li-zhong, 2002: 195.

Distribution: Vietnam; China.

13. Aphrodisium niisatoi Vives \& Bentanachs, 2007

Aphrodisium niisatoi Vives \& Bentanachs, 2007: 635.

Vives \& Bentanachs, 2007: 635.

Distribution: N. Vietnam (Vinh Phu); China.

14. Aphrodisium tokinea Pic, 1925

Aphrodisium tokinea Pic, 1925: 17.

Gressitt \& Rondon, 1970: 144.

Distribution: Vietnam.

15. Aphrodisium tricoloripes Pic, 1925

Aphrodisium tricoloripes Pic, 1925: 18.

Gressitt \& Rondon, 1970: 143.

Synonym: Aphrodosium (s.str.) tricoloripes Podany, 1971; Aphrodisium tricoloripes Breuning \& Itzinger, 1943.

Distribution: Vietnam; Laos.

16. Aromiella fruhstoferi Podany, 1971

Aromiella fruhstoferi Podany, 1971: 303.

Hua Li-zhong, 2002: 196.

Distribution: Vietnam; China.

17. Chelidonium argentatum (Dalman, 1817)

Cerambyx argentatum Dalman, 1817: Gressitt \& Rondon, 1970: 152.

Hua Li-zhong, 2002: 200.

Synonym: Chelidonium argentatum: Thomsom, 1864. 
Distribution: Vietnam; India; China; Myanmar; Laos.

18. Chelidonium binotaticolle Pic, 1937

Chelidonium binotaticolle Pic, 1937: Gressitt \& Rondon, 1970: 152.

Gressitt \& Rondon, 1970: 152.

Distribution: N. Vietnam; China; Laos.

19. Chelidonium herteli Podany, 1974

Chelidonium herteli Podany, 1974: Hua Lizhong, 2002: 200.

Hua Li-zhong, 2002: 200.

Distribution: Vietnam; China.

20. Chelidonium violaceimembris Gressitt \& Rondon, 1970

Chelidonium violaceimembris Gressitt \& Rondon, 1970: 151.

Gressitt \& Rondon, 1970: 151.

Distribution: Vietnam (Vinh Phuc, Cao Bang); China; Laos.

21. Chloridolum (Leontium) cyaneonotatum Pic, 1925

Chloridolum cyaneonotatum Pic, 1925: 18.

Gressitt \& Rondon, 1970: 174.

Distribution: N. Vietnam; China; Laos.

22. Chloridolum (Leontium) descarpentriesi Pic, 1949

Chloridolum descarpentriesi Pic, 1949: Gressitt \& Rondon, 1970: 172.

Gressitt \& Rondon, 1970: 172.

Distribution: N. Vietnam; Laos.

23. Chloridolum (Leontium) jeanvoinei Pic, 1932

Leontium lameerei var. jeanvoinei Pic, 1932: Gressitt \& Rondon, 1970: 175.

Gressitt \& Rondon, 1970: 175.

Distribution: Vietnam: N. Vietnam, S. Vietnam (Dak Lak, Dak Song, Kon Tum); China; Laos.

24. Chloridolum grossepunctatum Gressitt \& Rondon, 1970

Chloridolum grossepunctatum Gressitt \& Rondon, 1970: 170.
Vives \& Mei-ying Lin, 2013: 72.

Distribution: Vietnam (Vinh Phuc); China; Laos.

25. Chloridolum heyrovskyi Plavilstshikov, 1933

Chloridolum heyrovskyi Plavilstshikov, 1933: Gressitt \& Rondon, 1970: 168.

Gressitt \& Rondon, 1970: 168.

Distribution: N. Vietnam.

26. Chloridolum perlaetum (White, 1853)

Leontium perlaetum White, 1853: 161.

Hua Li-zhong, 2009: 294.

Distribution: Vietnam; India; China; Myanmar; Laos.

27. Chloridolum violaceicolle Pic, 1925

Chloridolum violaceicolle Pic, 1925: 19.

Gressitt \& Rondon, 1970: 168.

Distribution: Vietnam: Tonkin.

\section{Embrikstrandia unifasciata (Ritsema, 1897)}

Zonopterus unifasciatus Ritsema, 1897: Gressitt \& Rondon, 1970: 137.

Gressitt \& Rondon, 1970: 137.

Synonym: Embrik-Strandia unifasciata: Gressitt, 1940.

Distribution: S. Vietnam (Gia Lai); China; Laos, SW. India; Indonesia (Assam, Borneo).

29. Embrikstrandia distinca (Nonfried, 1891)

Embrikstrandia distinca (Nonfried, 1891): Gressitt \& Rondon, 1970: 136.

Gressitt \& Rondon, 1970: 136.

Distribution: Vietnam: Cochin; China; Myanmar; Laos.

30. Embrikstrandia inexpectata Podany, 1968

Embrikstrandia inexpectata Podany, 1968: Bentanachs, 2005: 6.

Bentanachs, 2005: 6.

Distribution: Vietnam; Myanmar.

31. Laosaphrodisium subplicatum (Pic, 1937)

Chelidonium gibbicolle v. subplicatum Pic, 1937: Vives \& Mei-ying Lin, 2013: 73. 
Vives \& Mei-ying Lin, 2013: 73.

Synonym: Aphrodisium subplicatum; Gressitt \& Rondon, 1970; Chelidonium subplicatum; Podany, 1974; Laosaphrodisium subplicatum; Bentanachs, 2012.

Distribution: Vietnam (Hoa Binh); China; Laos.

32. Pachyteria coomani Pic, 1943

Pachyteria coomani Pic, 1943: Gressitt \& Rondon, 1970: 139.

Hoang Vu Tru, Ta Huy Thinh, Cao Quynh Nga, 2011: 986 .

Distribution: Vietnam (Gia Lai).

33. Pachyteria diversipes Ritsema, 1890

Pachyteria diversipes Ritsema, 1890: 170.

Gressitt \& Rondon, 1970: 139.

Distribution: S. Vietnam.

34. Pachyteria semiplicata Pic, 1927

Pachyteria semiplicata Pic, 1927: Hua Lizhong, 2002: 222.

Hua Li-zhong, 2002: 222.

Distribution: Vietnam; China.

\section{Pachyteria similis Ritsema, 1890}

Pachyteria similis Ritsema, 1890: 171.

Gressitt \& Rondon, 1970: 139.

Distribution: S. Vietnam.

36. Polyzonus luteonotatus (Pic, 1928)

Chelidonium luteonotatum Pic, 1928: Gressitt \& Rondon, 1970: 162.

Gressitt \& Rondon, 1970: 162.

Synonym: Chelidonium luteonotatum var. plicatum Pic, 1932.

Distribution: N. Vietnam; Indo-China.

\section{Polyzonus obtusus Bates, 1879}

Polyzonus obtusus Bates, 1879: 413.

Gressitt \& Rondon, 1970: 162.

Synonym: Polyzonus obtusus var. reductus Pic, 1946; Polyzonus obtusus var. interuptus Pic, 1946.

Distribution: S. Vietnam; Laos; Cambodia; Myanmar.
38. Polyzonus prasinus (White, 1893)

Promeces prasinus White, 1893: Gahan, 1906: 219.

Gressitt \& Rondon, 1970: 155.

Synonym: Polyzonus prasinus: Gahan, 1906.

Distribution: Vietnam; China; India; Cambodia; Thailand.

39. Polyzonus saigonensis Bates, 1879

Polyzonus saigonensis Bates, 1879: 413.

Gressitt \& Rondon, 1970: 162.

Synonym: Polyzonus saigonensis var. semiviridis Pic, 1946.

Distribution: S. Vietnam; Laos; Myanmar; Malayxia.

40. Polyzonus similis Podany, 1980

Polyzonus (Polyzonus) similis Podany, 1980: Delahaye, 2012: 469.

Delahaye, 2012: 469.

Synonym: Polyzonus similis Bentanachs, 2012.

Distribution: Vietnam: Annam.

41. Schmidtiana violaceothoracica (Gressitt \& Rondon, 1970)

Pachyteria violaceothoracia Gressitt \& Rondon, 1970: 140-141.

Niisato, 1998b: 450.

Distribution: Vietnam (Vinh Phu); Laos.

42. Schwarzerium hasuoi Niisato et J. Bentanachs, 2012

Schwarzerium hasuoi Niisato et J. Bentanachs, 2012: 7.

Niisato \& Bentanachs, 2012: 7.

Distribution: Vietnam (Lao Cai).

Tribe Callidiopini Lacordaire, 1869: 360

43. Ceresium albomaculatum Pic, 1926

Ceresium albomaculatum Pic, 1926: Gressitt \& Rondon, 1970: 99.

Gressitt \& Rondon, 1970: 99.

Distribution: N. Vietnam; China; Laos.

44. Ceresium delauneyi Lameere, 1893 
Ceresium delauneyi Lameere, 1893: Gressitt \& Rondon, 1970: 96.

Gressitt \& Rondon, 1970: 96.

Distribution: Vietnam (Thua Thien-Hue, S. Vietnam).

45. Ceresium infranigrum Pic, 1937

Ceresium infranigrum Pic, 1937: Gressitt \& Rondon, 1970: 95.

Gressitt \& Rondon, 1970: 95.

Distribution: N. Vietnam.

46. Ceresium jeanvoinei Pic, 1932

Ceresium jeanvoinei Pic, 1932: Gressitt \& Rondon, 1970: 99.

Gressitt \& Rondon, 1970: 99.

Distribution: N. Vietnam; Laos.

47. Ceresium leuco sticticum White, 1855

Ceresium leucosticticum White, 1855: 245.

Hoang Vu Tru, Ta Huy Thinh, Cao Quynh Nga, 2011: 984.

Distribution: Vietnam (Gia Lai).

48. Ceresium ludificum Holzschuh, 1995

Ceresium ludificum Holzschuh, 1995: 20.

Holzschuh, 1995: 20.

Distribution: Vietnam (Tonkin, Hanoi).

49. Ceresium sinicum ornaticolle Pic, 1907

Ceresium ornaticollis Pic, 1907: Gressitt \& Rondon, 1970: 97.

Gressitt \& Rondon, 1970: 97.

Distribution: Vietnam; China; Laos.

50. Ceresium zeylanicum White, 1855

Ceresium zeylanicum White, 1855: 246.

Gressitt \& Rondon, 1970: 99.

Synonym: Ceresium sinicum ornaticolle: Plavilstshikov, 1931; Ceresium sinicum: Gressitt, 1942; Ceresium basilanum Picard, 1943.

Distribution: Vietnam (Da Lat); India; Srilanka; Miama; Laos; Indonesia (Java, Sumatra, Borneo); Philippines.

51. Examnes granulosus (Pic, 1931)
Ceresium granulosus Pic, 1931: Gressitt \& Rondon, 1970: 99.

Holzschuh, 2007: 210.

Distribution: Vietnam; Laos; Thailand.

52. Falsoibidion punctuosum Holzschuh, 2003

Falsoibidion punctuosum Holzschuh, 2003: 177

Holzschuh, 2003: 177.

Distribution: Vietnam (Ninh Binh).

53. Gelonaetha hirta (Fairmaire, 1850)

Stromatium hirtum Fairmaire, 1850: Gahan, 1906: 155 .

Hua Li-zhong, 2002: 209.

Synonym: Astrimus obscurus Sharp, 1878.

Distribution: Vietnam (Gia Lai); China; Myanmar; India; Srilanka; Cambodia; Thailand; Malaysia; Philippines.

54. Lasiophanes comosus Holzschuh, 1989

Lasiophanes comosus Holzschuh, 1989: 370.

Holzschuh, 1989: 370.

Distribution: Vietnam: Tonkin (Vinh Phuc).

55. Lasiophanes cristulatus Aurivillius, 1917

Lasiophanes cristulatus Aurivillius, 1917: Gressitt \& Rondon, 1970: 93.

Gressitt \& Rondon, 1970: 93.

Distribution: N. Vietnam; Laos.

\section{Salpinia laosensis Gressitt \& Rondon, 1970}

Salpinia laosensis Gressitt \& Rondon, 1970: 102-103.

Gressitt \& Rondon, 1970: 102-103.

Distribution: Vietnam (Vinh Phu, Thua ThienHue); Laos.

57. Stenygrinum quadrinotatum Bates, 1873

Stenygrinum quadrinotatum Bates, 1873: 154.

Hua Li-zhong, 2009: 324.

Distribution: Vietnam; Japan; Korea; China; Taiwan; Myanmar; India (Assam); Malaysia, Philippines; Indonesia (Kalimantan).

58. Stenodryas atripes (Pic, 1935) 
Stenodryas atripes Pic, 1935: Gressitt \& Rondon, 1970: 104.

Gressitt \& Rondon, 1970: 104.

Distribution: S. Vietnam.

59. Stenodryas bicoloripes (Pic, 1922)

Ibidiomimus bicoloripes Pic, 1922: Gressitt \& Rondon, 1970: 104.

Gressitt \& Rondon, 1970: 104.

Distribution: N. Vietnam; Laos.

60. Stenodryas inapicalis (Pic, 1922)

Stenodryas inapicalis Pic, 1922: Gressitt \& Rondon, 1970: 104.

Gressitt \& Rondon, 1970: 104.

Distribution: N. Vietnam.

Tribe Cerambycini Latreille, 1804: Gahan, 1906: 119

61. Aeolesthes (Pseudaeolesthes) aureopilosa Gressitt \& Rondon, 1970

Aeolesthes (Pseudaeolesthes) aureopilosa Gressitt \& Rondon, 1970: 63.

Hoang Vu Tru, Ta Huy Thinh, Cao Quynh Nga, 2011: 983 .

Distribution: Vietnam (Gia Lai).

62. Aeolesthes (Pseudaeolesthes) rufimembris Pic, 1923

Aeolesthes (Pseudaeolesthes) rufimembris Pic, 1923: 8.

Gressitt \& Rondon, 1970: 63.

Distribution: N. Vietnam,; Laos.

63. Aeolesthes (s.str.) holosericea (Fabricius, 1787)

Cerambyx holosericea Fabricius, 1787: Gahan, 1906: 127.

Ta Huy Thinh \& Hoang Vu Tru, 2005: 303.

Synonym: Aeolesthes holocericea: Gahan, 1906.

Distribution: Vietnam (Thua Thien-Hue); Laos; S. China; India, Sri Lanka; Thailand; Malaysia; Indonexia.

64. Aeolesthes aurifaber (White, 1853)
Hammaticherus aurifaber White, 1853: 128.

Hoang Vu Tru, Ta Huy Thinh, Cao Quynh Nga, 2011: 983.

Distribution: Vietnam (Gia Lai).

65. Cyriopalus wallacei Pascoe, 1866

Cyriopalus wallacei Pascoe, 1866: Gressitt \& Rondon, 1970: 59.

Ta Huy Thinh \& Hoang Vu Tru, 2005: 303.

Synonym: Cyriopalus wallacei: Wallacei, 1869.

Distribution: Vietnam (Thua Thien-Hue); Laos.

66. Derolus blaisei Pic, 1923

Derolus blaisei Pic, 1923: 8.

Gressitt \& Rondon, 1970: 74.

Distribution: Vietnam: N. Vietnam, S. Vietnam (Gia Lai).

67. Derolus griseonotatus Pic, 1923

Derolus griseonotatus Pic, 1923: 8.

Gressitt \& Rondon, 1970: 74.

Distribution: N. Vietnam.

68. Derolus volvulus (Fabricius, 1801)

Cerambyx volvulus Fabricius, 1801: Gressitt \& Rondon, 1970: 74.

Hua Li-zhong, 2002: 204.

Distribution: Vietnam; China (Hainan); Laos; India; Philippines; Indonesia (Java); Saudi Arabia.

69. Dialeges pauper Pascoe, 1856

Dialeges pauper Pascoe, 1856: 47.

Gressitt \& Rondon, 1970: 85.

Distribution: Vietnam (Kon Tum); India (Assam); E. Pakistan; Bangladesh; Myanmar; Laos; Thailand; Malaysia; Indonesia (Borneo).

70. Dialeges undulatus Gahan, 1891

Dialeges undulatus Gahan, 1891: 23.

Hoang Vu Tru, Ta Huy Thinh, Cao Quynh Nga, 2011: 984.

Distribution: Vietnam (Kon Tum, Gia Lai).

71. Diorthus cinereus (Fabricius, 1793)

Cerambyx cinereus Fabricius, 1793: Gressitt \& Rondon, 1970: 71. 
Hoang Vu Tru, Ta Huy Thinh, Cao Quynh Nga, 2011: 984 .

Synonym: Diorthus cinereus Lepesme, 1953; Taurotagus brevipennis Mateu, 1963; Diorthus (Diorthus) cinereus Gressitt \& Rondon, 1970.

Distribution: Vietnam (Kon Tum, Gia Lai).

72. Dymasius cos Holzschuh, 1998

Dymasius cos Holzschuh, 1998: 65.

Holzschuh, 1998: 65.

Distribution: Central Vietnam (Thua ThienHue), S. Vietnam.

73. Dymasius gracilicornis (Gressitt, 1951)

Zegriades gracilicornis Gressitt, 1951: 147-148.

Holzschuh, 2010: 137-225.

Distribution: N. Vietnam (Vinh Phuc); Laos; China.

\section{Dymasius verticosus Holzschuh, 2010}

Dymasius verticosus Holzschuh, 2010: 137-225.

Holzschuh, 2010: 137-225.

Distribution: Vietnam: Tonkin.

75. Falsoxeanodera maculata Pic, 1946

Falsoxeanodera maculata Pic, 1946: Hua Lizhong, 2002: 209.

Hua Li-zhong, 2002: 209.

Distribution: Vietnam; China.

76. Hoplocerambyx spinicornis (Newman, 1942)

Hammaticherus spinicornis Newman, 1842: Gahan, 1906: 131.

Gressitt \& Rondon, 1970: 70.

Distribution: Vietnam (Quang Nam, Kon Tum,

Gia Lai); Afghanistan; Pakistan; India, Myanmar, Laos, Malaysia.

77. Margites grisescens Pic, 1937

Margites grisescens Pic, 1937: Gressitt \& Rondon, 1970: 78.

Gressitt \& Rondon, 1970: 78.

Distribution: Vietnam; Laos.

78. Margites aggregatus Holzschuh, 1999

Margites aggregatus Holzschuh, 1999: 64.
Holzschuh, 1999: 64.

Distribution: S. Vietnam.

79. Massicus trilineatus (Pic, 1933)

Dymasius trilineatus Pic, 1933: 12.

Gressitt \& Rondon, 1970: 59.

Distribution: Vietnam: Tonkin, S. Vietnam (Quang Nam, Gia Lai); China; Taiwan; Laos.

80. Nadezhdiella fulvopubens (Pic, 1933)

Plocederus fulvopubens Pic, 1933: 27.

Holzschuh, 2005: 4.

Synonym: Nadezhdiella aurens Gressitt, 1937.

Nadezhdiella conica Chiang, 1942.

Distribution: Vietnam (Vinh Phuc); China, Laos, Thailand.

81. Neocerambyx vit alisi Pic, 1923

Neocerambyx vitalisi Pic, 1923: 12.

Gressitt \& Rondon, 1970: 58.

Distribution: S. Vietnam, Laos, Borneo, IndoChina.

82. Neocerambyx grandis Gahan, 1891

Neocerambyx grandis Gahan, 1891: 20.

Ta Huy Thinh \& Hoang Vu Tru, 2005: 302-304.

Distribution: Vietnam (Quang Tri).

83. Neocerambyx paris (Wood, 1821)

Cerambyx paris Wood, 1821: Gressitt \& Rondon, 1970: 58.

Ta Huy Thinh \& Hoang Vu Tru, 2005: 303.

Distribution: Vietnam (Quang Tri).

84. Plocaederus obesus Gahan, 1890

Plocaederus obesus Gahan, 1890: 51.

Ta Huy Thinh \& Hoang Vu Tru, 2005: 303.

Distribution: Vietnam (Quang Tri, Dak Lak, Dak Nong, Gia Lai); S. China; India (Assam); Myanmar (Andaman island); Laos; Thailand.

85. Plocaederus ruficornis (Newman, 1842)

Cerambyx ruficornis Newman, 1842: 245.

Hoang Vu Tru, Ta Huy Thinh, Cao Quynh Nga, 2011: 986.

Synonym: Cerambyx fulvicornis Guerin, 1843. 
Distribution: Vietnam (Kon Tum, Gia Lai, Dak Lak, Dak Nong); Laos.

86. Rhytidodera bowringii White, 1853

Rhytidodera bowringii White, 1853: 133.

Hoang Vu Tru, Ta Huy Thinh, Cao Quynh Nga, 2011: 987 .

Distribution: Vietnam (Gia Lai).

87. Rhytidodera grandis Thomson, 1865

Rhytidodera grandis Thomson, 1865: Gressitt \& Rondon, 1970: 88.

Hoang Vu Tru, Ta Huy Thinh, Cao Quynh Nga, 2011: 987 .

Distribution: Vietnam (Gia Lai); Laos.

88. Rhytidodera integra Kolbe, 1886

Rhytidodera integra Kolbe, 1886: Gressitt \& Rondon, 1970: 88.

Ta Huy Thinh \& Hoang Vu Tru, 2005: 303.

Distribution: Vietnam (Thua Thien-Hue, Quang Nam, Gia Lai).

\section{Trachylophus acutulus Holzschuh, 2009}

Trachylophus acutulus Holzschuh, 2009: 267358.

Holzschuh, 2009: 267-358.

Distribution: Vietnam (Vinh Phuc).

90. Trirachys orientalis Hope, 1841

Trirachys orientalis Hope, 1841: Gressitt \& Rondon, 1970: 68.

Ta Huy Thinh \& Hoang Vu Tru, 2005: 303

Synonym: Trirachys formosana Schwarzer, 1925.

Distribution: Vietnam (Quang Nam); Japan (Kyushu); S. China, Taiwan; Laos.

91. Xoanodera (Lajoyeia) vitticollis Gahan, 1891

Xoanodera vitticollis Gahan, 1891: 32.

Gressitt \& Rondon, 1970: 91.

Synonym: Lajoyeia interrupta Pic, 1933.

Distribution: Vietnam (Kon Tum); Laos; Indonesia (Borneo).

92. Xoanodera interrupta (Pic, 1933)
Lajoyeia interrupta Pic, 1933: 10.

Heffern, 2005: 17.

Distribution: Vietnam; Borneo; Sumatra.

93. Xoanodera maculata Schwarzer, 1925

Xoanodera maculata Schwarzer, 1925: 21.

Hoang Vu Tru, Ta Huy Thinh, Cao Quynh Nga, 2011: 987 .

Distribution: Vietnam (Gia Lai).

94. Xoanodera regularis Gahan, 1890

Xoanodera regularis Gahan, 1890: 52-53.

Ta Huy Thinh, Hoang Vu Tru, 2005: 303.

Distribution: Vietnam (Thua Thien-Hue, Quang Nam, Kon Tum).

95. Zatrephus longicornis Pic, 1930

Zatrephus longicornis Pic, 1930: 15.

Gressitt \& Rondon, 1970: 88.

Distribution: Vietnam: Tonkin; Laos; China.

Tribe Cleomenini Lacordaire, 1869: 97

96. Artimpaza argenteonotata Pic, 1922

Artimpaza argenteonotata Pic, 1922: Gressitt \& Rondon, 1970: 291.

Gressitt \& Rondon, 1970: 291.

Distribution: Vietnam (Tonkin); China; Laos.

97. Artimpaza curtelilneata (Pic, 1922)

Falsodebilia curtelilneata Pic, 1922: Gressitt \& Rondon, 1970: 291.

Gressitt \& Rondon, 1970: 291.

Distribution: N. Vietnam; SE. China; Laos.

98. Cleomenes nigricollis Fairmaire, 1895

Cleomenes nigricollis Fairmaire, 1895: Gressitt \& Rondon, 1970: 298.

Gressitt \& Rondon, 1970: 298.

Distribution: Vietnam: Tonkin, S. Vietnam (Ban Me Thuot, Dak Nong, Gia Lai); China; Laos.

99. Cleomenes tenuipes Gressitt, 1939

Cleomenes tenuipes Gressitt, 1939: 106.

Hua Li-zhong, 2002: 202.

Distribution: Vietnam; Laos; China; India; Malaysia. 
100. Dere affinis macilenta Gressitt, 1940

Dere macilenta Gressitt, 1940: 87.

Hua Li-zhong, 2002: 202.

Distribution: Vietnam; China.

101. Dere contigua Holzschuh, 1995

Dere contigua Holzschuh, 1995: 64.

Holzschuh, 1995: 64.

Distribution: Vietnam: Tonkin (Vinh Phuc).

102. Dere deversaria Holzschuh, 1995

Dere deversaria Holzschuh, 1995: 64.

Holzschuh, 1995: 64.

Distribution: Vietnam: Tonkin (Vinh Phuc).

103. Kurarua elongaticollis (Pic, 1932)

Debilissa elongaticollis Pic, 1932: Gressitt \& Rondon, 1970: 301.

Gressitt \& Rondon, 1970: 301.

Distribution: N. Vietnam; Laos.

104. Kurarua longula Holzschuh, 1999

Kurarua longula Holzschuh, 1999: 64.

Holzschuh, 1999: 64.

Distribution: N. Vietnam.

105. Paramimistena ovicollis Holzschuh, 1991

Paramimistena ovicollis Holzschuh, 1991: 5354.

Holzschuh, 1991:53-54.

Distribution: N. Vietnam (Lang Son).

106. Paramimistena subglabra subglabra Gressitt \& Rondon, 1970: 306

Paramimistena subglabra Gressitt \& Rondon, 1970: 306.

Niisato, 2009: 360.

Distribution: Vietnam: Tonkin; India; Myanmar Laos.

Tribe Clytini Mulsant, 1839: Gahan, 1906 : 239

107. Amamiclytus dembickyi Holzschuh, 1991

Amamiclytus dembickyi Holzschuh, 1991: 46.

Holzschuh, 1991: 46.

Distribution: N. Vietnam: Tonkin (Vinh Phuc).
108. Clytus bellus Holzschuh, 1998

Clytus bellus Holzschuh, 1998: 65.

Holzschuh, 1998: 65.

Distribution: N. Vietnam.

109. Cyrtoclytus dalatensis Niisato et Kusakabe, 2009

Cyrtoclytus dalatensis Niisato et Kusakabe, 2009: 232.

Niisato et al., 2009: 232.

Distribution: S. Vietnam (Da Lat).

110. Cyrtoclytus takakuwai Niisato et Kusakabe, 2009

Cyrtoclytus takakuwai Niisato et Kusakabe, 2009: 228.

Niisato et al., 2009: 232.

Distribution: Vietnam (Vinh Phuc).

111. Chlorophorus annularis (Fabricius, 1787)

Callidium annulare Fabricius, 1787: Gressitt \& Rondon, 1970: 221.

Gressitt \& Rondon, 1970: 221.

Distribution: Vietnam (Quang Tri, Kon Tum); Japan (Ryukyu); Korea; S. China; Taiwan; India; Sri Lanka; Nepal; Myanmar; Laos; Cambodia; Thailand; Malaysia; Indonexia; Philippines; New Guinea.

112. Chlorophorus arciferus (Chevrolat, 1863)

Amauraesthes arciferus Chevrolat, 1863: 330.

Hoang Vu Tru, Ta Huy Thinh, Cao Quynh Nga, 2011: 984.

Synonym: Clytanthus verbasci rectefasciatus Pic, 1937; Chlorophorus varius subsp. pieli Gressitt, 1951.

Distribution: Vietnam (Dak Lak, Dak Nong, Kon Tum, Gia Lai).

\section{Chlorophorus brevenotatus Pic, 1922}

Chlorophorus brevenotatus Pic, 1922: Gressitt \& Rondon, 1970: 228.

Gressitt \& Rondon, 1970: 228.

Distribution: Vietnam: Tonkin; Laos. 
114. Chlorophorus coniperda Holzschuh, 1992

Chlorophorus coniperda Holzschuh, 1992: 27.

Holzschuh, 1992: 27.

Distribution: S. Vietnam (Da Lat).

115. Chlorophorus grandipes Pic, 1943

Chlorophorus grandipes Pic, 1943: 2.

Gressitt \& Rondon, 1970: 228.

Distribution: S. Vietnam; Laos.

116. Chlorophorus japonicus (Chevrolat, 1863)

Chlorophorus japonicus (Chevrolat, 1863: 298)

Hua Li-zhong, 2002: 201.

Distribution: Vietnam; Japan; Korea; China.

117. Chlorophorus jendeki Holzschuh, 1992

Chlorophorus jendeki Holzschuh, 1992: 22.

Holzschuh, 1992: 22.

Distribution: Vietnam: Tonkin (Vinh Phuc).

118. Chlorophorus micheli Pic, 1943

Chlorophorus micheli Pic, 1943: Hua Li-zhong, 2002 : 201.

Hua Li-zhong, 2002 : 201.

Distribution: Vietnam; China.

119. Chlorophorus pinguis Holzschuh, 1992

Chlorophorus pinguis Holzschuh, 1992: 21.

Holzschuh, 1992: 21.

Distribution: Vietnam: Tonkin (Hoang Lien Son, $\mathrm{Sa} \mathrm{Pa})$.

120. Chlorophorus punctiger tamdaoensis Holzschuh, 1992

Chlorophorus punctiger tamdaoensis

Holzschuh, 1992: 22.

Holzschuh, 1992: 22.

Distribution: Vietnam: Tonkin (Vinh Phuc).

121. Chlorophorus quatuordecimmaculatus (Chevrolat, 1863)

Anthoboscus 14-maculatus Chevrolat, 1863: 295.

Gressitt \& Rondon, 1970: 228.

Synonym: Cholorophorus guerryi: Gressitt, 1951.
Distribution: N. Vietnam; Laos; S. China; India. 122. Chlorophorus reductus Pic, 1922

Chlorophorus reductus Pic, 1922: 13.

Gressitt \& Rondon, 1970: 228.

Distribution: Vietnam: N. Vietnam, S. Vietnam (Dak Lak, Dak Nong, Kon Tum, Gia Lai); S. China; Laos.

123. Chlorophorus viticis Gressitt \& Rondon, 1970

Chlorophorus viticis Gressitt \& Rondon, 1970: 225.

Hua Li-zhong, 2002: 202.

Distribution: Vietnam; Laos; China.

124. Chlorophorus vulpinus Holzschuh, 1992

Chlorophorus vulpinus Holzschuh, 1992: 21.

Holzschuh, 1992: 21.

Distribution: Vietnam: Tonkin (Hoang Lien Son, $\mathrm{Sa} \mathrm{Pa})$.

125. Demonax alcanor Gressitt \& Rondon, 1970

Demonax alcanor Gressitt \& Rondon, 1970: 270.

Hoang Vu Tru, Ta Huy Thinh, Cao Quynh Nga, 2011: 982-988.

Synonym: Demonax kuroikoi Niisato, 1990.

Distribution: Vietnam (Dak Nong).

126. Demonax binotatithorax Pic, 1927

Demonax binotatithorax Pic, 1927: Gressitt \& Rondon, 1970: 275.

Gressitt \& Rondon, 1970: 275.

Distribution: N. Vietnam; Laos.

127. Demonax bowringi (Pascoe, 1859)

Clytus bowringii Pascoe, 1859: 28.

Gressitt \& Rondon, 1970: 275.

Synonym: Demonax bowringii: Aurivillius, 1912; Demonax breveapicalis Pic, 1927.

Distribution: S. Vietnam (Gia Lai); China; Laos.

128. Demonax brevespinosus Pic, 1926 
Demonax brevespinosus Pic, 1926: Gressitt \& Rondon, 1970: 275.

Gressitt \& Rondon, 1970: 275.

Distribution: N. Vietnam; Laos.

129. Demonax diversefasciatus Pic, 1920

Demonax diversefasciatus Pic, 1920: Gressitt \& Rondon, 1970: 265.

Thinh \& Tru, 2005: 303.

Distribution: Vietnam (Thua Thien-Hue); SW. China; Laos.

\section{Demonax maximus Pic, 1922}

Demonax maximus Pic, 1922: Gressitt \& Rondon, 1970: 265.

Gressitt \& Rondon, 1970: 265.

Distribution: N. Vietnam; China; Laos.

131. Demonax musivus Pascoe, 1869

Demonax musivus Pascoe, 1869: 630.

Hayashi, 1976: 167-209.

Distribution: S. Vietnam; Laos; Thailand; Indonesia (Sumatra, Borneo).

\section{Demonax offensus Holzschuh, 1992}

Demonax offensus Holzschuh, 1992: 38.

Holzschuh, 1992: 38.

Distribution: Vietnam: Tonkin (Vinh Phuc).

133. Demonax perspicuus Holzschuh, 1992

Demonax perspicuus Holzschuh, 1992: 39.

Holzschuh, 1992: 39.

Distribution: Vietnam: Tonkin (Vinh Phuc).

134. Demonax piliger Holzschuh, 1992: 39

Demonax piliger Holzschuh, 1992: 39.

Holzschuh, 1992: 39.

Distribution: Vietnam: Tonkin (Vinh Phuc).

135. Demonax semiluctuosus (White, 1855)

Clytus semiluctuosus White, 1855: 283.

Gressitt \& Rondon, 1970: 263.

Synonym: Demonax albobifasciatus Pic, 1920.

Distribution: N. Vietnam; India; Myanmar; Malaysia; Indonexia; Indochina.

136. Demonax tristiculus (Fairmaire, 1895)
Piazomias tristiculus Fairmaire, 1888: Hua Lizhong, 2002: 204 .

Hua Li-zhong, 2002: 204.

Distribution: Vietnam, China.

137. Perissus atronotatus Pic, 1937

Perissus atronotatus Pic, 1937: Gressitt \& Rondon, 1970: 217.

Gressitt \& Rondon, 1970: 217.

Distribution: Vietnam: North Central; Laos.

138. Perissus biluteofasciatus Pic, 1918

Perissus biluteofasciatus Pic, 1918: Gressitt \& Rondon, 1970: 215.

Gressitt \& Rondon, 1970: 215.

Distribution: N. Vietnam; Laos.

139. Perissus elongatus Pic, 1937

Perissus elongatus Pic, 1937: Gressitt \& Rondon, 1970: 215.

Gressitt \& Rondon, 1970: 215.

Distribution: Vietnam: North Central; Laos.

140. Perissus mutabilis Gahan, 1894

Perissus mutabilis Gahan, 1894 : 23.

Hoang Vu Tru, Ta Huy Thinh, Cao Quynh Nga, 2011: 986 .

Distribution: Vietnam (Dak Nong).

141. Perissus mutabilis obscuricolor Pic, 1937

Perissus mutabilis obscuricolor Pic, 1937:

Gressitt \& Rondon, 1970: 215.

Gressitt \& Rondon, 1970: 215.

Distribution: N. Vietnam; Laos.

142. Perissus mutabilis vitalisi Pic, 1923

Perissus multabilis var. vitalisi Pic, 1923:

Gressitt \& Rondon, 1970: 2.

Gressitt \& Rondon, 1970: 215.

Distribution: N. Vietnam, Laos.

143. Perissus pacholatkoi Holzschuh, 1992

Perissus pacholatkoi Holzschuh, 1992: 19.

Holzschuh, 1992: 19.

Distribution: Vietnam (Vinh Phuc, Hoa Binh).

144. Rhaphuma binhensis (Pic, 1922) 
Chlorophorus binhensis Pic, 1922: 13.

Hua Li-zhong, 2009: 319.

Synonym: Rhaphuma binhensis: Gressitt, 1951.

Distribution: Vietnam; China; Laos.

145. Rhaphuma brodskyi Holzschuh, 1992

Rhaphuma brodskyi Holzschuh, 1992: 28.

Holzschuh, 1992: 28.

Distribution: Vietnam (Vinh Phuc).

146. Rhaphuma excisa Holzschuh, 1992

Rhaphuma excisa Holzschuh, 1992: 36.

Holzschuh, 1992: 36.

Distribution: Vietnam (Vinh Phuc).

147. Rhaphuma horsfieldi (White, 1855)

Clytus horsfieldi White, 1855: 284.

Gressitt \& Rondon, 1970: 241.

Synonym: Rhaphuma horsfieldi: Gahan, 1906; Rhaphuma horsfieldi var. laoensis Pic, 1923.

Distribution: N. Vietnam; SW. China; Laos; India; Myanmar.

148. Rhaphuma improba Holzschuh, 1992

Rhaphuma improba Holzschuh, 1992: 32.

Holzschuh, 1992: 32.

Distribution: Vietnam: Tonkin (Vinh Phu).

149. Rhaphuma innotata Pic, 1927

Rhaphuma innotata Pic, 1927: Gressitt \& Rondon, 1970: 234.

Gressitt \& Rondon, 1970: 234.

Distribution: Vietnam (Tonkin: Vinh Phu).

150. Rhaphuma maceki Holzschuh, 1992

Rhaphuma maceki Holzschuh, 1992: 29.

Holzschuh, 1992: 29.

Distribution: Vietnam: Tonkin (Vinh Phuc).

151. Rhaphuma manipurensis vietnamica Holzschuh, 1992

Rhaphuma manipurensis vietnamica Holzschuh, 1992: 29.

Holzschuh, 1992: 29.

Distribution: Vietnam: Tonkin (Vinh Phuc).
152. Rhaphuma patkaina Gahan, 1906

Rhaphuma patkaina Gahan, 1906: 272.

Hua Li-zhong, 2002: 230.

Distribution: Vietnam; China; India; Laos.

153. Rhaphuma placida Pascoe, 1858

Rhaphuma placida Pascoe, 1858: 240.

Hoang Vu Tru, Ta Huy Thinh, Cao Quynh Nga, 2011: 987.

Distribution: Vietnam (Kon Tum).

154. Rhaphuma rufobasalis Pic, 1924

Rhaphuma rufobasalis Pic, 1924: Gressitt \& Rondon, 1970: 234.

Gressitt \& Rondon, 1970: 234.

Distribution: Vietnam: Tonkin.

155. Rhaphuma rybniceki Holzschuh, 1992

Rhaphuma rybniceki Holzschuh, 1992: 30.

Holzschuh, 1992: 30.

Distribution: Vietnam: Tonkin (Vinh Phuc).

156. Rhaphuma rybniceki Holzschuh, 1992

Rhaphuma rybniceki Holzschuh, 1992: 30.

Holzschuh, 1992: 30.

Distribution: Vietnam: Tonkin (Vinh Phuc).

157. Rhaphuma strnadi Holzschuh, 1992

Rhaphuma strnadi Holzschuh, 1992: 31.

Holzschuh, 1992: 31.

Distribution: Vietnam: Tonkin (Vinh Phuc).

158. Sclethrus amoerus (Gory, 1833)

Clytus amoenus Gory, 1833: Gressitt \& Rondon, 1970: 279.

Gressitt \& Rondon, 1970: 279.

Distribution: N. Vietnam; Laos; China; Indonesia (Kalimantan); Singapo; Sumatra; Borneo; Philippines.

159. Sclethrus stenocylindrus Fairmaire, 1895

Sclethrus stenocylindrus Fairmaire, 1895: 184.

Chang \& Niisato, 2009: 237-279.

Synonym: Sclethrus amoenus: Gressitt \& Rondon, 1970. 
Distribution: Vietnam (Vinh Phuc, Son La, Quang Nam); China; Myanmar; Laos; Thailand; Indochina.

160. Xylotrechus buqueti (Castelnau et Gory, 1841)

Clytus buqueti Castelnau et Gory, 1841: Hua Li-zhong, 2002: 236.

Hua Li-zhong, 2002: 236.

Synonym: Xylotrechus buqueti: Chevrolat, 1863.

Distribution: Vietnam; China; India; Laos; Thailand; Indonesia.

161. Xylotrechus clavicornis Pic, 1927

Xylotrechus clavicornis Pic, 1927: Gressitt \& Rondon, 1970: 194.

Gressitt \& Rondon, 1970: 194.

Distribution: N. Vietnam.

162. Xylotrechus contusus Holzschuh, 2003

Xylotrechus contusus Holzschuh, 2003: 201.

Holzschuh, 2003: 201.

Distribution: Vietnam: Tonkin (Vinh Phuc).

163. Xylotrechus curtithorax Pic, 1922

Xylotrechus curtithorax Pic, 1922: 12.

Gressitt \& Rondon, 1970: 201.

Distribution: N. Vietnam; Laos.

164. Xylotrechus dalatensis Pic, 1928

Xylotrechus dalatensis Pic, 1928: Gressitt \& Rondon, 1970: 197.

Gressitt \& Rondon, 1970: 197.

Distribution: Vietnam (Sai Gon).

165. Xylotrechus diversenotatus Pic, 1930

Xylotrechus diversenotatus Pic, 1930: Gressitt \& Rondon, 1970: 198.

Gressitt \& Rondon, 1970: 198.

Distribution: Vietnam (Tonkin).

166. Xylotrechus diversepubens Pic, 1930

Xylotrechus diversepubens Pic, 1930: Gressitt \& Rondon, 1970: 195.

Gressitt \& Rondon, 1970: 195.

Distribution: Vietnam: Tonkin.
167. Xylotrechus diversesignatus magdelainei Pic, 1937

Xylotrechus diversesignatus var. magdelainei Pic, 1937: Gressitt \& Rondon, 1970: 200.

Gressitt \& Rondon, 1970: 200.

Distribution: S. Vietnam; Laos; S-W. China; Thailand.

168. Xylotrechus imperfectus Chevrolat, 1863

Xylotrechus imperfectus Chevrolat, 1863: 313.

Gressitt \& Rondon, 1970:201.

Synonym: Xylotrechus salvazai Pic, 1928.

Distribution: S. Vietnam; Laos; Malaysia; Indonesia (Sumatra, Borneo).

169. Xylotrechus innotatithorax Pic, 1927

Xylotrechus innotatithorax Pic, 1927: Gressitt \& Rondon, 1970: 195.

Gressitt \& Rondon, 1970: 195.

Distribution: Vietnam: Tonkin.

170. Xylotrechus longithorax Pic, 1923

Xylotrechus longithorax Pic, 1923: 13.

Gressitt \& Rondon, 1970: 203.

Distribution: N. Vietnam; China; Laos.

\section{Xylotrechus nodieri Pic, 1933}

Xylotrechus nodieri Pic, 1933: Gressitt \& Rondon, 1970: 196.

Gressitt \& Rondon, 1970: 196.

Distribution: Vietnam: Tonkin.

172. Xylotrechus olexai Holzschuh, 1992

Xylotrechus olexai Holzschuh, 1992: 18.

Holzschuh, 1992: 18.

Distribution: Vietnam: Tonkin (Vinh Phuc).

173. Xylotrechus paulocarinatus Pic, 1930

Xylotrechus paulocarinatus Pic, 1930: Gressitt \& Rondon, 1970: 1.

Gressitt \& Rondon, 1970: 196.

Distribution: Vietnam: Tonkin.

174. Xylotrechus quadripes Chevrolat, 1863

Xylotrechus quadripes Chevrolat, 1863: 315.

Gressitt \& Rondon, 1970: 200. 
Distribution: Vietnam; China; India; Myanmar; Laos; Thailand.

175. Xylotrechus rufobasalis Pic, 1937

Xylotrechus rufobasalis Pic, 1937: Gressitt \& Rondon, 1970: 199.

Gressitt \& Rondon, 1970: 199.

Distribution: Vietnam: Annam; Laos.

176. Xylotrechus semimarginatus Pic, 1922

Xylotrechus semimarginatus Pic, 1922: Gressitt \& Rondon, 1970: 195.

Gressitt \& Rondon, 1970: 195.

Distribution: Vietnam (Saigon).

177. Xylotrechus signaticollis Pic, 1922

Xylotrechus signaticollis Pic, 1922: Gressitt \& Rondon, 1970: 196.

Gressitt \& Rondon, 1970: 196.

Distribution: Vietnam (Tonkin).

178. Xylotrechus trimaculatus Pic, 1922

Xylotrechus trimaculatus Pic, 1922: 12.

Gressitt \& Rondon, 1970: 195.

Distribution: N. Vietnam.

179. Xylotrechus uniannulatus Pic, 1925

Xylotrechus uniannulatus Pic, 1925: 20.

Gressitt \& Rondon, 1970: 197.

Distribution: Vietnam: Tonkin.

180. Xylotrechus unicarinatus Pic, 1917

Xylotrechus unicarinatus Pic, 1917: Gressitt \& Rondon, 1970: 201.

Gressitt \& Rondon, 1970: 201.

Distribution: Vietnam: Tonkin; Laos.

Tribe Hesperophanini Mulsant, 1839: Gahan, 1906: 109

\section{Gnatholea eburifera Thomson, 1861}

Gnatholea eburifera Thomson, 1861: 375.

Gressitt \& Rondon, 1970: 52.

Distribution: Vietnam (Buon Me Thuot, Dak Song, Kon Tum, Gia Lai); S. China; India (Assam); Myanmar; Laos; Cambodia; Thailand; Malaysia; Indinesia (Kalimantan).
182. Stromatium longicorne (Newman, 1842)

Arhopalus longicornis Newman, 1842: 246.

Hoang Vu Tru, Ta Huy Thinh, Cao Quynh Nga, 2011: 987.

Synonym: Stromatium longicorne: Gahan, 1906.

Distribution: Vietnam (Kon Tum, Gia Lai); Japan (Ryukyu); S. China; Taiwan; E. India (Assam); Myanmar; Laos; SE. Asia; Philippines.

183. Zoodes fulguratus Gahan, 1906

Zoodes fulguratus Gahan, 1906: 117.

Hua Li-zhong, 2002: 237.

Distribution: Vietnam; Laos; China; Myanmar.

184. Zoodes japonicus Hayashi, 1963

Zoodes japonicus Hayashi, 1963: Niisato, 2000: 86.

Niisato, 2000: 86.

Distribution: Vietnam (Vinh Phuc); SW. Japan.

Tribe Methiini Thomson, 1860: 364

185. Comusia apicalis (Pic, 1922)

Chapaon apicalis Pic, 1922: 10.

Gressitt \& Rondon, 1970: 49.

Distribution: Vietnam: N. Vietnam, S. Vietnam (Kon Tum, Gia Lai); Laos.

186. Comusia atra (Pic, 1922: 23)

Hypomares ater Pic, 1922: Gressitt \& Rondon, 1970: 49.

Gressitt \& Rondon, 1970: 49.

Distribution: N. Vietnam; Laos.

187. Comusia bicoloricornis (Pic, 1926)

Chapaon rubicoloricorne Pic, 1926: Gressitt \& Rondon, 1970: 49.

Gressitt \& Rondon, 1970: 49.

Distribution: N. Vietnam; Laos.

188. Comusia rufa (Pic, 1922)

Chapaon rufum Pic, 1922: 12.

Gressitt \& Rondon, 1970: 49.

Distribution: N. Vietnam; Laos. 
189. Comusia ruficornis (Pic, 1946)

Chapaon rufum var ruficorne Pic, 1946: Gressitt \& Rondon, 1970: 49.

Gressitt \& Rondon, 1970: 49.

Distribution: Vietnam: Tonkin; Laos.

190. Hyphus apicalis Pascoe, 1869

Hyphus apicalis Pascoe, 1869: 505.

Heffern, 2005: 1-104.

Distribution: Vietnam; Laos; Thailand; Indonesia (Borneo).

191. Leptoxenus ramosanus Holzschuh, 2007

Leptoxenus ramosanus Holzschuh, 2007: 205.

Holzschuh, 2007: 205.

Distribution: S. Vietnam (An Khe, Buon Luoi).

192. Noserius tiabialis Pascoe, 1857

Noserius tiabialis Pascoe, 1857: 95.

Gressitt \& Rondon, 1970: 47.

Distribution: S. Vietnam (Kon Tum, Gia Lai);

Laos; Cambodia; Malaya; Borneo; Myanmar;

Java; Singapore.

193. Oemospila callidioides Gressitt \& Rondon, 1970

Oemospila callidioides Gressitt \& Rondon, 1970: 46.

Heffern, 2011: 9.

Distribution: Vietnam; Laos; Indonesia (Borneo).

194. Tetraommatus insignis Gahan, 1894

Tetraommatus insignis Gahan, 1894: Gressitt \& Rondon, 1970: 44.

Gressitt \& Rondon, 1970: 44.

Synonym: Tetraommatus spinicollis Pic, 1922.

Distribution: N. Vietnam; S. Myanmar (Andamans); Laos; Thailand.

\section{Xystrocera festiva Thomson, 1861}

Xystrocera festiva Thomson, 1861: 251.

Gressitt \& Rondon, 1970: 51.

Distribution: Vietnam: Tonkin, Central Vietnam (Quang Tri), S. Vietnam (Gia Lai); China; India (Assam); Myanmar; Laos; Indonesia (Sumatra, Borneo, Java).
196. Xystrocera globosa (Olivier, 1795)

Cerambyx globorus Olivier, 1795: Gressitt \& Rondon, 1970: 51.

Gressitt \& Rondon, 1970: 51.

Synonym: Xystrocera globosa var. medioviticollis Breuning, 1957.

Distribution: Vietnam: N. Vietnam, S. Vietnam (Kon Tum, Gia Lai); Japan; Korea; China; Taiwan; Laos; Philippines; S. Asia; Celebes; Pacific islands; Madagascar; Mauritius; Egypt.

197. Xystrocera imperfectus Chevrolat, 1863

Xystrocera imperfectus Chevrolat, 1863: 313.

Makihara et al., 2002: 192.

Distribution: S. Vietnam; Laos; Indonesia (Borneo, Java).

Tribe Molorchini Mulsant, 1863: Gressitt \& Rondon, 1970: 109

198. Epania bicoloricornis Pic, 1950

Epania bicoloricornis Pic, 1950: Gressitt \& Rondon, 1970: 112.

Gressitt \& Rondon, 1970: 112.

Distribution: Vietnam (Sai Gon).

199. Epania kasaharai Niisato, 2002

Epania kasaharai Niisato, 2002: 348.

Niisato, 2002: 348.

Distribution: N. Vietnam (Vinh Phuc).

200. Epania lineola Pic, 1933

Epania lineola Pic, 1933: Gressitt \& Rondon, 1970: 112.

Gressitt \& Rondon, 1970: 112.

Distribution: Vietnam: Tonkin.

201. Epania minuta Pic, 1935

Epania minuta Pic, 1935: Gressitt \& Rondon, 1970: 112.

Gressitt \& Rondon, 1970: 112.

Distribution: Vietnam: Tonkin.

202. Epania paulloides Niisato, 2002

Epania paulloides Niisato, 2002: 352.

Niisato, 2002: 352.

Distribution: N. Vietnam (Vinh Phuc). 


\section{Epania ruficollis Pic, 1922}

Epania ruficollis Pic, 1922: 36.

Niisato et al., 1996: 147-158.

Distribution: N. Vietnam (Vinh Phuc); Laos; N. Thailand

204. Epania vietnamica Niisato et A. Saito, 1996

Epania vietnamica Niisato et A. Saito, 1996: 152.

Niisato et A. Saito, 1996: 152.

Distribution: N. Vietnam (Vinh Phuc).

205. Euchlanis atricornis Pic, 1940

Microdebilissa atricornis Pic, 1940: Gressitt \& Rondon, 1970: 12.

Gressitt \& Rondon, 1970: 129.

Distribution: Vietnam; China.

206. Euchlanis minutus Pic, 1927

Euchlanis minutus Pic, 1927: Gressitt \& Rondon, 1970: 129.

Gressitt \& Rondon, 1970: 129.

Distribution: Vietnam (Gia Lai).

207. Euchlanis subviridis Gressitt \& Rondon, 1970

Euchlanis subviridis Gressitt \& Rondon, 1970: 129.

Gressitt \& Rondon, 1970: 129.

Distribution: Vietnam; Laos.

208. Glaphyra yui masatakai Niisato et A. Saito, 1996

Glaphyra yui masatakai Niisato et A. Saito, 1996: 155.

Niisato et A. Saito, 1996: 155.

Distribution: N. Vietnam (Lai Chau).

209. Kunbir elongaticollis (Pic, 1932)

Debilissa elongaticollis Pic, 1932: Gressitt \& Rondon, 1970: 127.

Gressitt \& Rondon, 1970: 127.

Distribution: Vietnam: Tonkin.

210. Kunbir rufoflavida (Fairmaire, 1924)
Debilissa rufoflavida Fairmaire, 1924: Gressitt \& Rondon, 1970: 127.

Gressitt \& Rondon, 1970: 127.

Distribution: Vietnam: Tonkin.

211. Kunbir testacea (Pic, 1924)

Debilissa testacea Pic, 1924: Hua Li-zhong, 2002: 212.

Hua Li-zhong, 2002: 212.

Distribution: Vietnam.

212. Merionoeda (Macromolorchus) miranda Holzschuh, 1989

Merionoeda (Macromolorchus) miranda Holzschuh, 1989: 361-390.

Holzschuh, 1989: 361-390.

Distribution: Vietnam: Tonkin (Hoa Binh).

213. Merionoeda baliosmerion Gressitt \& Rondon, 1970

Merionoeda baliosmerion Gressitt \& Rondon, 1970: 122.

Hoang Vu Tru, Ta Huy Thinh, Cao Quynh Nga, 2011: 985.

Distribution: Vietnam (Kon Tum).

214. Merionoeda jeanvoinei Pic, 1933

Merionoeda jeanvoinei Pic, 1933: Gressitt \& Rondon, 1970: 117.

Gressitt \& Rondon, 1970: 117.

Distribution: Vietnam: Tonkin.

215. Molorchoepania barbieri (Pic, 1949)

Epania (Molorchoepania) barbieri Pic, 1949: 9.

Gressitt \& Rondon, 1970: 112.

Synonym: Molorchoepania: Hayashi, 1974.

Distribution: S. Vietnam; Laos; Thailand.

216. Molorchoepania coomani (Pic, 1940)

Epania coomani Pic, 1940: 112.

Gressitt \& Rondon, 1970: 112.

Synonym: Epania (Molorchoepania) coomani: Gressitt \& Rondon, 1970; Molorchoepania coomani: Hayashi, 1974.

Distribution: N. Vietnam; Laos. 
Tribe Mythodini Lacordaire, 1869: Gahan, 1906: 182

217. Phyodexia concinna Pascoe, 1871

Phyodexia concinna Pascoe, 1871: 273.

Hua Li-zhong, 2002: 224.

Distribution: Vietnam; China; India; Myanmar; Bhutan; Laos.

Tribe Obriini Mulsant, 1839: Gressitt \& Rondon, 1970: 105

218. Obrium posticum saigonense Pic, 1933

Obrium posticum Gahan var. saigonensis Pic, 1933: 9.

Gressitt \& Rondon, 1970: 107.

Distribution: S. Vietnam; Laos.

219. Obrium akikoae Niisato, 1998

Obrium akikoae Niisato, 1998: 267-276.

Niisato, 1998: 267-276.

Distribution: N. Vietnam (Son La).

220. Obrium elongatum Niisato, 1998

Obrium elongatum Niisato, 1998: 267-276.

Niisato, 1998: 267-276.

Distribution: N. Vietnam (Lai Chau).

221. Obrium huae Niisato, 1998

Obrium huae Niisato, 1998: 267-276.

Niisato, 1998: 267-276.

Distribution: N. Vietnam (Cao Bang).

222. Obrium coomani Pic, 1927

Obrium coomani Pic, 1927: Gressitt \& Rondon, 1970: 107.

Gressitt \& Rondon, 1970: 107.

Distribution: N. Vietnam.

223. Uenobrium laosicum (Gressitt \& Rondon, 1970)

Obrium laosicum Gressitt \& Rondon, 1970: 107

Niisato, 2006: 211.

Distribution: N. Vietnam; Laos; China.

224. Falsobrium minutum Pic, 1931

Falsobrium minutum Pic, 1931: Gressitt \& Rondon, 1970: 108.
Gressitt \& Rondon, 1970: 108.

Distribution: N. Vietnam; Laos.

225. Stenhomalus cephalotes Pic, 1928

Stenhomalus cephalotes Pic, 1928: 28.

Gressitt \& Rondon, 1970: 108.

Distribution: N. Vietnam; China; Laos, Indonesia (Borneo).

226. Stenhomalus nigerrimus Holzschuh, 1999

Stenhomalus nigerrimus Holzschuh, 1999: 64.

Holzschuh, 1999: 64.

Distribution: S. Vietnam (Da Lat).

227. Longipalpus apicalis (Pic, 1926)

Ibidionidum apicale Pic, 1926: Gressitt \& Rondon, 1970: 108.

Gressitt \& Rondon, 1970: 108.

Distribution: N. Vietnam (Vinh Phuc); Laos; Borneo.

228. Ibidionidum jelineki Holzschuh, 1991

Ibidionidum jelineki Holzschuh, 1991: 41-42.

Holzschuh, 1991: 41-42.

Distribution: Vietnam (Vinh Phuc); China.

Tribe Prothemini Lacordaire, 1869: Gahan, 1906: 232

229. Prothema signata Pascoe, 1856

Prothema signata Pascoe, 1856: 43.

Gressitt \& Rondon, 1970: 134.

Distribution: Vietnam: N. Vietnam, S. Vietnam

(Gia Lai); S. China; Laos.

230. Prothema aurata cariniscapa (Gressitt, 1937)

Prothema cariniscapa Gressitt, 1937: 451-452.

Hua Li-zhong, 2002: 226.

Distribution: Vietnam; China.

231. Prothema aurata Gahan, 1906

Prothema aurata Gahan, 1906: 235.

Ta Huy Thinh \& Hoang Vu Tru, 2005: 303.

Distribution: Vietnam (Quang Nam, Gia Lai).

Tribe Purpuricenini Gahan, 1906: 183 
232. Bunothorax takasagoensis (Kano, 1933: 278)

Sternoplistes takasagoensis Kano, 1933: 278.

Niisato, 2004: 442.

Synonym: Bunothorax takasagoensis: Gressitt, 1936.

Distribution: N. Vietnam (Vinh Phuc); Laos; Taiwan.

233. Euryphagus miniatus (Fairmaire, 1904)

Purpuricenus miniatus Fairmaire, 1904: 145.

Hua Li-zhong, 2002: 208.

Distribution: Vietnam; China.

234. Euryphagus lundii (Fabricius, 1792)

Cerambyx lundii Fabricius, 1792.

Hayashi, 1976: 28.

Synonym: Euryphagus lundii: Gahan, 1906.

Distribution: Vietnam; India; Myamar; Laos; Cambodia; Thailand; Singapore; Malaysia; Indonesia (Sumatra, Borneo).

Tribe Pyrestini Lacordaire, 1869: Gahan, 1906: 224

235. Erythrus laticornis Fairm, 1895

Erythrus laticornis Fairm, 1895: Gressitt \& Rondon, 1970: 179.

Gressitt \& Rondon, 1970: 179.

Distribution: N. Vietnam; Laos.

236. Erythrus apicalis Pic, 1922

Erythrus apicalis Pic, 1922: Gressitt \& Rondon, 1970: 182.

Gressitt \& Rondon, 1970: 182.

Distribution: N. Vietnam; Laos.

237. Erythrus atripennis Pic, 1926

Erythrus atripennis Pic, 1926: Gressitt \& Rondon, 1970: 182.

Gressitt \& Rondon, 1970: 182.

Distribution: Vietnam (Gia Lai); Laos.

238. Pachylocerus sulcatus Brongniart, 1892

Pachylocerus sulcatus Brongniart, 1892: Hua Li-zhong, 2002: 222.

Hua Li-zhong, 2002: 222.
Distribution: Vietnam (Gia Lai); S. China; Taiwan; India (Assam); Myanmar; Laos; Thailand.

239. Plutonesthes melanoderes Holzschuh, 1995

Plutonesthes melanoderes Holzschuh, 1995: 64.

Holzschuh, 1995: 64.

Distribution: Vietnam (Tonkin: Vinh Phuc).

240. Pyrestes rufipes Pic, 1923

Pyrestes rufipes Pic, 1923: 13.

Gressitt \& Rondon, 1970: 184.

Distribution: N. Vietnam; Laos.

241. Pyrestes rudis Holzschuh, 2005

Pyrestes rudis Holzschuh, 2005: 41.

Holzschuh, 2005: 41.

Distribution: N. Vietnam (Vinh Phuc).

Tribe Phoracanthini Lacordaire, 1869: 298

242. Allotraeus asiaticus (Schwarzer, 1925)

Neosphaerion asiaticum Schwarzer, 1925: 22.

Hua Li-zhong, 2002: 192.

Synonym: Allotaraeus asiaticus: Matsushita, 1933.

Distribution: Vietnam; Japan; China; Taiwan.

243. Allotraeus gracillimus (Mitono, 1947)

Pseudallotraeus gracillimus Mitono, 1947: 24.

Hua Li-zhong, 2009: 286.

Synonym: Allotraeus gracillimus: Gressitt, 1951.

Distribution: Vietnam; China; Taiwan.

244. Allotraeus (Nysina) orientalis (White, 1853)

Sphaerion orientalis White, 1853: 110.

Hoang Vu Tru, Ta Huy Thinh, Cao Quynh Nga, 2011: 983.

Synonym: Nyphasia orientalis Gahan, 1906; Pseudallotraeus atripes Pic, 1923.

Distribution: Vietnam (Gia Lai).

245. Allotraeus rubriventris Gressitt, 1937

Allotraeus rubriventris Gressitt, 1937: 90. 
Hua Li-zhong, 2002: 192.

Distribution: Vietnam; China.

246. Nyphasia pascoei Lacordaire, 1869

Nyphasia pascoei Lacordaire, 1869: 309.

Hoang Vu Tru, Ta Huy Thinh, Cao Quynh Nga, 2011: 986.

Distribution: Vietnam (Gia Lai).

Tribe Rosaliini Fairmaire, 1864: Gahan, 1906: 175

247. Acrocyrtidus argenteofasciatus (Pic 1903)

Mausaridaeus argenteofasciatus Pic, 1903: 30.

Gressitt \& Rondon, 1970: 186.

Distribution: Vietnam; China.

248. Acrocyrtidus attenuatus (Pic, 1927)

Acrocyrtidus attenuatus (Pic, 1927): Gressitt \& Rondon, 1970: 186.

Gressitt \& Rondon, 1970: 186.

Distribution: N. Vietnam.

249. Acrocyrtidus avarus Holzschuh, 1979

Acrocyrtidus avarus Holzschuh, 1979: 293-294.

Thinh \& Tru, 2005: 983.

Distribution: Vietnam (Gia Lai).

250. Acrocyrtidus diversinotatus (Pic, 1903)

Mausaridaeus diversinotatus Pic, 1903: Gressitt \& Rondon, 1970: 186.

Gressitt \& Rondon, 1970: 186.

Distribution: Vietnam; China.

251. Rosalia decempunctata (Westwood, 1848)

Purpuricenus 10-punctata Westwood, 1848: Gressitt \& Rondon, 1970: 190.

Hoang Vu Tru, Ta Huy Thinh, Cao Quynh Nga, 2011: 987.

Distribution: Vietnam (Gia Lai).

252. Rosalia (s.str.) lameerei Brongniart, 1890

Rosalia lameerei Brongniart, 1890: Gressitt \& Rondon, 1970: 190.

Hua Li-zhong, 2002: 231.
Synonym: Rosalia lameerei var.quadrinotata Dayrem, 1935; Rosalia lameerei var.unireducta Pic, 1937.

Distribution: Vietnam.

Tribe Tillomorphini Lacordaire, 1869: 88 253. Epipedocera atra Pic, 1937

Epipedocera atra Pic, 1937: 7.

Gressitt \& Rondon, 1970: 284.

Distribution: Vietnam: Annam; China; Laos.

254. Epipedocera effusa Holzschuh, 1999

Epipedocera effusa Holzschuh, 1999: 64.

Holzschuh, 1999: 64.

Distribution: Vietnam: Tonkin.

Tribe Thraniini Gahan, 1906: 236

255. Thranius formosanus atripennis Pic, 1946: 53

Thranius atripennis Pic, 1946: Gressitt \& Rondon, 1970: 191.

Gressitt \& Rondon, 1970: 191.

Distribution: N. Vietnam; Laos.

256. Thranius irregularis Pic, 1927

Th ranius irregularis Pic, 1927: Gressitt \& Rondon, 1970: 191.

Gressitt \& Rondon, 1970: 191.

Distribution: N. Vietnam.

257. Thranius multinotatus Pic, 1922

Thranius multinotatus Pic, 1922: 22.

Gressitt \& Rondon, 1970: 193.

Distribution: N. Vietnam; China; Laos.

258. Thranius ornatus Gressitt \& Rondon, 1970

Thranius ornatus Gressitt \& Rondon, 1970: 191.

Hoang Vu Tru, Ta Huy Thinh, Cao Quynh Nga, 2011: 987.

Distribution: Vietnam (Kon Tum).

259. Thranius signatus Schwarzer, 1925

Thranius signatus Schwarzer, 1925: 25. 
Hua Li-zhong, 2002: 235.

Distribution: Vietnam; China; Laos.

Acknowledgments: I thank to IDEA WILD for providing quipments in surveys and study.

\section{REFERENCES}

1. Abang F., 2013. Cerambycidae in Sarawak. http://www.arbec.com.my/cerambycinae /. Acessessed 8.5.2014

2. Aurivillius C., 1911. Coleoptera Longicornia II. Arkiv for Zoologi. Band, 7(9): 44p.

3. Aurivillius C., 1912. Coleoptorum Catalogus, pars 39 [vol. 22], Cerambycidae: Cerambycinae. Berlin, W. Junk \& S. Schenkling. 574 pp.

4. Bates H. W., 1873. On the longicorn Coleoptera of Japan. The Annals and Magazine of Natural History, 12(4): 148156. London.

5. Bates H. W., 1879. New Genera and Species of Callichrominae (Coleoptera Longicornia). Cistula Entomologica, 2: 395 419. London.

6. Bentanachs J., 2005. Une nouvelle espece du genre Embrik-Strandia Plavilstshikov, 1931 (Coleoptera, Cerambycidae, Callichromatini). Museu de Zoologia de Barcelona. Les Cahiers Magallanes, 50: 6p.

7. Bentanachs J., Vives E., Jhel P., 2008. Revison du genre Anubis Thomson, 1864. Collection systematique, 17: 65 .

8. Bily S., Mehl O., 1989. Longhorn Beetles (Coleoptera: Cerambycidae) of Fenoscandia and Denmark. Fauna Ent. Scand., 22. Leiden. 203pp.

9. Bouchard P., Bousquet Y., Davies A. E., Alonso-Zarazaga M. A., Lawrence J. F., Lyal C. H. C., Newton A. F., Reid C. A. M., Schmitt M., Slipinski S. A., \& Smith A. B. T., 2011. Family-group names in Coleoptera (Insecta). Zookeys, 88: 1-972.

10. Bousquet Y., Daniel J. H., Bouchard P., \& E. H. Nearns, 2009. Catalogue of familygroup names Cerambycidae (Coleoptera). Zootaxa, 2321: 1-80.
11. Chevrolat L. A. A., 1863. Clytides d'Asie e d'Océanie. Mémoires de la Société Royale des Sciences de Liège, 18(4): 253-350.

12. Dauber D., 1995. Xylotrechus buquetti (Laporte \& Gory, 1836) and related species (Coleoptera: Cerambycidae: Cerambycinae). G. it. Ent., 7: 301-316.

13. Delahaye N., 2012. Catalogue of Cerambycinae.

14. Http://www.prioninae.org/Data/Catalogue $\%$ 20Cerambycinae). Acessessed 8.5.2014

15. Fabricius J. C., $1775 . \quad$ Systema Entomologiae, sistens insectorum classes, ordines, genera, species, adiectis synonymis, locis, descriptionibus, observationibus. Officina Libraria Kortii, Flensburgi et Lipsiae. 832 pp.

16. Fairmaire L., 1895. Deuxième note sur quelques Coléoptères des environs de LangSong. Annales de la Société Entomologique de Belgique, Bruxelles. 39: 173-190.

17. Fairmaire L., 1896. Beetles of the boreal India, China \& Malaysia. Notes from the Leyden Museum, 18: 81-129.

18. Gahan C. J., 1890. Descriptions of new species of Longicornia from India and Ceylon. The Annals and Magazine of Natural History, London. (6) 5(25): 48-66. 1 pl. 8 fig.

19. Gahan C. J., 1891. Notes on longicorn Coleoptera of the group Cerambycinae with description of new genera and species. The Annals and Magazine of Natural History, London. (6) 7(37): 19-34.

20. Gahan C. J., 1894. A list of the longicorn Coleoptera collected by Signor Fea in Myanmar and the adjoining regions with descriptions of the new genera and species. Viaggio di Leonardo Fea in Birmania e regioni vicine. Annali del Museo Civico di Storia Naturale di Genova. (2a) 14(44): 5104.

21. Gahan C. J., 1906. The Fauna of British India including Ceylon and Myanmar. Coleoptera. Vol. I (Cerambycidae). London. 329pp. 
22. Gressitt J. L., 1939. A collection of longicorn beetles from T'ien-mu Shan, East China (Coleoptera: Cerambycidae). Notes sur l'Entomologie Chinoise, 6(4): 81-133.

23. Gressitt J. L., 1940. The longicorn beetles of Hainan Island. Philippine Journal of Science. Manila, 72(1/2): 1-239.

24. Gressitt J. L., 1951. Longicorn beetles of China. Longicornia vol. II. Paul Lechevalier, Paris. 677pp.

25. Gressitt J. L., Rondon J. A., 1970. Cerambycid-beetles of Laos. Pacific Insects Monograph. Bishop Museum, Honolulu, 24: 1-314.

26. Han Chang-do, Niisato T., 2009. Clytine Beetles of the Genus Sclethrus Newman (Coleoptera, Cerambycidae). Spec. Bull. Jpn. Soc. Coleopterol., Tokyo, 7: 237-279.

27. Hayashi M., 1976. On some longicorn beetles from Borneo and Malaya, chiefly from the entomological result of Dr. M. Hayashi's S. E. Asian Expedition in 1970 (Col: Cerambycidar), 167-209.

28. Hayashi M., 1976. On some longicorn beetles from Malaya descriptions of six new species (Col: Ceram.). Bull. Japan Ent. Academ, 9(2): 24-41.

29. Hayashi M., 1981. The Cerambycidae (Coleoptera) of Nepal collected by the Kyushu Unverstiy scientific Expedition. Esakia, 17: 183-200.

30. Heffern D. J., Ozdikmen H., 2007. Nomenclatural change for Oriental longhorn beetles (Coleoptera; Cerambycidae). Munis Entomology and Zoology, 2(1): 59-62.

31. Heffern D. J., 2011. Catalog and bibliography of longhorned beetles from Borneo (Coleoptera, Cerambycidae). Electronic Version, 1: 106

32. Heffern D. J., 2005. Catalog and Bibliography of Longhorned Beetles from Borneo (Coleoptera: Cerambycidae). http://www.zin.ru/animalia/Coleoptera/pdf/b orneo_catalog_electronic_version_20051.pdf. 102pp.

33. Hoang Vu Tru, Ta Huy Thinh, 2006. Spreading of the Longhorn (Cerambycidae,
Coleoptera) in the Middle part of Cetral Vietnam. The scientific conference on forward to agronomy management technology for development of sustainable agriculture in Vietnam, Hanoi, 543-550 (in Vietnamese, summary in English).

34. Hoang Vu Tru, Ta Huy Thinh, Cao Quynh Nga, 2009. Distribution of the Longhorn beetles (Cerambycidae, Coleoptera) in the Northern Truong Son range passing through Thanh Hoa, Nghe An and $\mathrm{Ha}$ Tinh provinces. Proceeding of the $3^{\text {rd }}$ national scientific conference on Ecology and Biological Resources. Agriculture Publishing House, Hanoi: 394-400 (in Vietnamese, summary in English).

35. Hoang Vu Tru, Ta Huy Thinh, Cao Quynh Nga, 2011. Result of the survey on longhorn beetles (Cerambycidae, Coleoptera) along the Ho Chi Minh road through Tay Nguyen highland. Proceeding of the $4^{\text {nd }}$ national scientific conference on Ecology and Biological Resources, Agriculture Publishing House, Hanoi (ISSN 18594425). 982-988 (in Vietnamese, summary in English).

36. Holzschuh C., 1989. Beschreibung von 20 neuen Bockkafern aus Thailand, Vietnam und Taiwan (Coleoptera, Cerambycidae). Entomologica Basiliensia, 13: 361-390.

37. Holzschuh C., 1991. Neue Bockkäfer aus Asien II. 63 neue Bockkäfer aus Asien, vorwiegend aus China und Thailand (Coleoptera: Disteniidae und Cerambycidae). FBVA Berichte, Schriftenreihe der Forstlichen Bundesversuchsanstalt. Vol. 60. Wien, Austria. 80 pp., 80 ill.

38. Holzschuh C., 1992. 57 neue Bockkafer aus Asien, vorwiegend aus China, Thailand und Vietnam (Coleoptera, Cerambycidae). Forstlicen Bundesversuchsanstalt. 63p.

39. Holzschuh C., 1995. Description of 65 new longhorned beetles from europe and asia, mostly from Thailand and China (Coleoptera: Disteniidae and Cerambycidae). FBVA-Berichte, 84: 64p. 
40. Holzschuh C., 1998. Description of 68 new longhorned beetles from asia, mostly from Thailand and China (Coleoptera: Disteniidae and Cerambycidae). FBVABerichte 107: 65p.

41. Holzschuh C., 1999. Description of 71 new longhorned beetles from Asia, mostly from China, Laos, Thailand and India (Coleoptera: Cerambycidae). FBVABerichte 110: 64p

42. Holzschuh C., 2003. Description of 72 new longhorned beetles from Asia, mostly from China, Laos, Thailand and India (Coleoptera: Cerambycidae). Entomologica Basiliensia, 25: 147-241

43. Holzschuh C., 2005. Description of new longhorned beetles from SE Asia and Borneo (Coleoptera: Cerambycidae). Les Cahiers Magellanes, 46: 41p

44. Holzschuh C., 2007. Description of 80 new longhorned beetles from Orientalis and Paleartic, mostly from China, Laos and Borneo (Coleoptera: Cerambycidae). Entomologica Basiliensia et Collectionis Frey, 29: 177-286

45. Holzschuh C., 2009. Description of 59 new longhorned beetles from Gattungen, Orientalis and Paleartic, mostly from China, Laos and Borneo (Coleoptera: Cerambycidae). Entomologica Basiliensia et Collectionis Frey, 31: 267-358

46. Holzschuh C., 2010. Description of 66 new longhorned beetles from Gattungen and Orientalis, mostly from China, Laos and Thailand (Coleoptera: Cerambycidae). Entomologica Basiliensia et Collectionis Frey, 32: 137-225

47. Hoppe P., Lameere M., 1919. Traite D'Entomologie Indochinoise. Cerambycidae. Impr. Minsang, Hanoi: 141-148.

48. Hua Li-zhong, 2002. List of Chinese Insects. Vol. II. Family Cerambycidae. Zhongshan (Sun Yat-sen) University Press: 189-237.

49. Hua Li-zhong, Hajime N., Saemulson G. A., \& Lingafelter S. W., 2009. Iconography of
Chinese Longicorn Beetles (1406 species) in Color. Series Publication of Museum of Biology. Sun Yat-sen University Press. 475p.

50. Hudepohl K. E., 1990. The longhorn beetles of the Philippines, Part II. Entomofauna: Zeitschrift für Entomologie, 11(3/1-2): 45102, 42 fig.

51. Huang J.-H., Zhou S.-Y., Chen B., 2006. Review of Chinese species of the genus Embrikstrandia Plavilstshikov, 1931 (Coleoptera: Cerambycidae: Cerambycinae) with description of a new species. Zootaxa, 1340: 57-68.

52. Lacordaire J. T., 1869. Histoire Naturelle des Insectes. Genera des Coleopteres, ou expose methodique et critique de tous les genres proposes jusqu'ici dans cet ordre d'insects. Paris. Librairie Encyclopedique de Roret., 8(1): 1-552.

53. Lacordaire J. T., 1872. Histoire Naturelle des Insectes. Genera des Coleopteres, ou expose methodique et critique de tous les genres proposes jusqu'ici dans cet ordre d'insects. IX. Deuzieme partie. Longicornes. Paris: 441-931.

54. Linnaeus C., 1758. Systema naturae per regna tria naturae secundum classes, ordines, genera, species cum characteribus, differentiis, synonymis, locis. Tomus I. Editio Decima, reformata. Laurentii Salvii, Holmiae, 1(10): 824 pp.

55. Makihara H., Noerdjito W. A., Sugiarto, 2002. Longicorn Beetles from Gunung Halimun National Park, West Java, Indonesia from 1997-2002 (Coleoptera, Disteniidae and Cerambycidae). Bulletin of FFPRI, 1(384): 189-223.

56. Mai Phu Quy, Tran Thi Lai, Tran Thi Bich Lan, 1981. The survey of animals in North Vietnam. Family Longhorned-beetles (Cerambycidae, Coleoptera). Science and Technology Publishing House, Hanoi: 131137 (in Vietnamese, summary in English).

57. Newman E., 1842. Cerambycitum insularum manillarum Dom. Cuming 
captorum enumeratio digesta. The Entomologist, 1(15): 243-248. London.

58. Niisato T., Saito A., 1996. Taxonomic notes on the Molorchine Beetles (Coleoptera, Cerambycidae) from northern Vietnam, with descriptions of two new taxa. Elytra, Tokyo, 24(1): 147-158.

59. Niisato T., 2002. Two new Epania (Coleoptera, Cerambycidae) from Mt. Tam Dao, northern Vietnam. Elytra, Tokyo, 30(2): 348-357.

60. Niisato T., 2009. The genus Paramimistena (Coleoptera, Cerambycidae) from Xieng Khouang Province of Central Laos with a Description of a new species. Jpn. J. syst. Ent., 15(2): 355-360.

61. Niisato T., Bentanachs J., 2012. A new Schwarzerium (Coleoptera, Cerambycidae) from North Vietnam. Elytra, Tokyo, New Series, 2(1): 7-11.

62. Niisato T., Wen-I Chou, Kusakabe Y., 2009. Taxonomic notes on the genus Cyrtocrylus (Coleoptera, Cerambycidae) from China and Indochina. Spec. Bull. Jpn. Soc. Coleopterol, 7: 221-235. Tokyo.

63. Niisato T., 1990. Contribution towards the Knowledge of the cerambycid fauna (Coleoptera, Cerambycidae) of Thailand. Elytra, Tokyo, 18(1): 109-128.

64. Niisato T., 1998. Additional account for Longipalpus apicalis (Coleoptera, Cerambycidae). Jpn. J. syst. Ent., 4(2): 301302.

65. Niisato T., 1998. Systematic Position of Pachyteria violaceothoracica (Coleoptera, Cerambycidae), with a New Locality Record. Elytra, Tokyo, 26(2): 450.

66. Niisato T., 1998. Three new Obrium (Coleoptera, Cerambycidae) from Northern Vietnam. Jpn. J. syst. Ent., 4(2): 267-276.

67. Niisato T., 2000. Occurrence of Zoodes japonicus (Coleoptera, Cerambycidae) in Northern Vietnam. Elytra, Tokyo, 28(1): 86

68. Niisato T., 2002. Epania opaca (Coleoptera, Cerambycidae) and its New Relative with a
Brief Note on the genus Molorchoepania. Elytra, Tokyo, 30(1): 235-246.

69. Niisato T., 2002. New locality of Salpinia laosensis (Coleoptera, Cerambycidae), with a note on its Systematic Position. Elytra, Tokyo, 30(1): 262.

70. Niisato T., 2002. Two new Epania (Coleoptera, Cerambycidae) from Mt. Tam Dao, Northern Vietnam. Elytra, Tokyo, 30(2): 348-357.

71. Niisato T., 2004. Occurrence of Bunothorax takasagonesis (Coleoptera, Cerambycidae) in Eastern Indochina. Elytra, Tokyo, 32(2): 442.

72. Niisato T., 2006. A new Obriine Genus Uenobrium (Coleoptera, Cerambycidae) and ít Components. Elytra, Tokyo, 34(1): 207221.

73. Niisato T., 2009. The genus Paramimistena (Coleoptera, Cerambycidae) from Xieng Khouang Province of central Laos, with a Descriptin of a new species. Jpn, J. syst. Ent, 15(2): 355-360.

74. Niisato T., 2011. A revision of the genus Amamiclytus Ohbayashi from Taiwan and the Ryukyu Islands (Coleoptera, Cerambycidae). Zookeys, 118: 19-52.

75. Pascoe F. P., 1856. Descriptions of new genera and species of Asiatic longicorn Coleoptera. The Transactions of the Entomological Society of London 4(2): 42 50.1 pl. (XVI).

76. Pascoe F. P., 1857. On new genera and species of longicorn Coleoptera. Part II. The Transactions of the Entomological Society of London, 4(2): 89-112. 2 pls. (XXIIXXIII).

77. Pascoe F. P., 1858. On new genera and species of longicorn Coleoptera. Part III. The Transactions of the Entomological Society of London, 4(2): 236-266. 2 pls. (XXV-XXVI).

78. Pascoe F. P., 1859. On new genera and species of Longicorn Coleoptera. Part IV. The Transactions of the Entomological Society of London, 5(2): 12-61. 1 pl. (II). 
79. Pascoe F. P., 1864. Longicornia Malayana; or, a Descriptive Catalogue of the Species of the three Longicorn Families Lamiidae, Cerambycidae and Prionidae collected by Mr. A. R. Wallace in the Malay Archipelago. (part I). The Transactions of the Entomological Society of London, 1(3): $1-96$.

80. Pascoe F. P., 1865. Longicornia Malayana; or, a Descriptive Catalogue of the Species of the three Longicorn Families Lamiidae, Cerambycidae and Prionidae collected by Mr. A. R. Wallace in the Malay Archipelago. (Part II). The Transactions of the Entomological Society of London, 2(3): 97-224.

81. Pascoe F. P., 1866. Longicornia Malayana; or, a Descriptive Catalogue of the Species of the three Longicorn Families Lamiidae, Cerambycidae and Prionidae collected by Mr. A. R. Wallace in the Malay Archipelago. (Part III). The Transactions of the Entomological Society of London, 3(3): 225-336.

82. Pascoe F. P., 1867. Longicornia Malayana; or, a Descriptive Catalogue of the Species of the three Longicorn Families Lamiidae, Cerambycidae and Prionidae collected by Mr. A. R. Wallace in the Malay Archipelago. (Part IV). The Transactions of the Entomological Society of London, 3(3): 337-464.

83. Pascoe F. P., 1868. Longicornia Malayana; or, a descriptive catalogue of the species of the three longicorn families Lamiidae, Cerambycidae and Prionidae collected by Mr. A. R. Wallace in the Malay Archipelago. (Part V). The Transactions of the Entomological Society of London, 3(3): 465-498.

84. Pascoe F. P., 1869. Longicornia Malayana; or, a descriptive catalogue of the species of the three longicorn families Lamiidae, Cerambycidae and Prionidae collected by Mr. A. R. Wallace in the Malay Archipelago. (Part VI). The Transactions of the Entomological Society of London, 3(3): 499-552.
85. Pascoe F. P., 1869. Longicornia Malayana; or, a descriptive catalogue of the species of the three longicorn families Lamiidae, Cerambycidae and Prionidae collected by Mr. A. R. Wallace in the Malay Archipelago. The Transactions of the Entomological Society of London, 3(3): 1739.

86. Pic M., 1904. Descriptions of various longicorns Europe and Asia. Material used for the study of Longicorns, 5(1): 7-9.

87. Pic M., 1908. New longicorns of southern China. Material used for the study of Longicorns, 7(1): 14-18.

88. Pic M., 1911. Longicorns of China in part new. Material used for the study of Longicorns, 8(1): 19-21.

89. Pic M., 1922. Nouveautés diverses. Mélanges Exotico-Entomologiques. Moulins, 35: 1-32.

90. Pic M., 1923. Nouveautés diverses. Mélanges Exotico-Entomologiques. Moulins, 38: 1-32.

91. Pic M., 1925. Nouveautés diverses. Mélanges Exotico-Entomologiques. Moulins, 44: 1-32.

92. Pic M., 1933. Nouveautés diverses. Mélanges Exotico-Entomologiques. Moulins, 62: 1-36.

93. Plant Protection Research Institute, 1976. Result of the survey of insect (1967-1968), Family Cerambycidae. Agriculture Publishing House, Hanoi, 213-229.

94. Podany C., 1971. Studien über Callichromini der palaearktischen und orientalischen region (II). Entomologische Abhandlungen Staatliches Museum für Tierkunde in Dresden, 38(8): 253-313.

95. Podany C., 1980. Studien über Callichromini der palaearktischen und orientalischen region (V) (Coleoptera, Cerambycidae). Entomologische Abhandlungen Staatliches Museum für Tierkunde in Dresden, 43(10): 219-256.

96. Ritsema C., 1890. On some species of the genus Pachyteria from the old 
collection of Thomson. Notes from the Leyden Museum, 12: 163-173.

97. Schwarzer B., 1925. Sauters FormosaAusbeute (Cerambycidae. Col.). (Subfamilie Cerambycinae). Entomologische Blätter. Zeitschrift für Biologie und Systematik der Käfer. Berlin \& Krefeld, 21: 20-30.

98. Ta Huy Thinh, Hoang Vu Tru, 2005. List of long-horn collected on Truongson moutains from Quang Tri, Thua Thien-Hue and Quang Nam province. Proceedings, The 2005th National Conference on Life Sciences. Science and Techniques Publishing House, Hanoi, 302-304 (in Vietnamese, summary in English).

99. Thomson J., 1860. Essai d'une classification de la famille des cérambycides et matériaux pour servir à une monographie de cette famille. Paris. 396 pp.

100. Thomson J., 1861. Essai d'une classification de la famille des cérambycides et matériaux pour servir à une monographie de cette famille. Paris. 396 pp.

101. Vitali F., 2007. About the taxonomic status of some species of the genus
Aeolesthes Gahan, 1890 (Coleoptera, Cerambycidae). Entomapeiron (N. S.), 1(3): 65-80.

102. Vitalis de Salvaza R., 1919. Traité d'entomologie indochinoise. Cerambycidae. Impr. Minsang, Hanoi, 139-162.

103. Vives E., Bentanachs J., 2007. Notes on Asian Callichromatini (I) description of one new species of the genus Aphrodisium Thomson, 1864. (Cleoptera, Cerambycidae). Lambillionea CVII, 4: 635-638.

104. Vives E., Mei-ying Lin, 2013. One new and seven newly recorded Callichromatini species from China (Coleoptera, Cerambycidae, Cerambycinae). Zookyes, 275: 67-75.

105. White A., 1853. Catalogue of coleopterous insects in the collections of the British museum, pt VII. Longicornia I. pp. 1-174. pl. 1-4. London.

106. White A., 1855. Catalogue of coleopterous insects in the collection of the British Museum, pt. VIII. Longicornia II. pp. 175-412. pl. 5-10.Zhou Wenyi, 2008. Colored Illustrations of Longicorn Beetles of Taiwan. 408pp. 


\title{
DANH LỤC CÁC LOÀI XÉN TÓC PHÂN HỌ Cerambycinae (Coleoptera: Cerambycidae) Ở VIẸT NAM
}

\author{
Cao Thị Quỳnh Nga, Khuất Đăng Long
}

Viện Sinh thái và Tài nguyên sinh vật, Viện Hàn lâm KH \& CN Việt Nam

\section{TÓM TẮT}

Cerambycinae là một trong các phân họ lớn thuộc họ Xén tóc Cerambicidae, sâu non của chúng sống trong thân cây đang còn sống hoặc cây đã chết khô, rất nhiều loài là những đối tượng gây hại cho cây trồng và cây rừng. Tổng số loài thuộc phân họ Cerambycinae đã biết trên thế giới ước tính tới 20.000 loài, chúng có mặt ở rất nhiều sinh cảnh từ gần bờ biển cho đến độ cao tới hơn $4.000 \mathrm{~m}$.

Bài báo này lần đầu tiên đưa ra danh lục đầy đủ để tra cứu 259 loài xén tóc thuộc 88 giống và 18 tộc của phân họ Cerambicinae thuộc khu hệ xén tóc ở Việt Nam, đồng thời cung cấp những thông tin về phân bố của các loài ở Việt Nam và trên thế giới đã được các tác giả trong và ngoài nước thống kê cho đến thời điểm hiện nay.

Trong số 18 tộc, 7 tộc có số loài nhiều nhất gồm: Clytini với 74 loài, Callichromini: 41, Cerambycini: 35, Molorchini: 19, Callidiopini: 18, Methiini: 13 và Cleomenini: 11 loài; còn lại 11 tộc có số loài ít hơn 10 loài.

Tù khóa: Danh lục, khu hệ, phân bố, phân họ, xén tóc.

Ngày nhận bài: 23-1-2014 